\title{
Dynamic Relationship of Industrial Structure Change and Economic Fluctuations: Evidence from Sichuan, China
}

\author{
Ying Feng ${ }^{1}$,' Dongmei $\mathrm{Li}^{1} \&$ Yanni Long ${ }^{1}$ \\ ${ }^{1}$ College of Management, Sichuan Agricultural University, Chengdu City, China \\ Correspondence: Ying Feng, College of Management, Sichuan Agricultural University, Chengdu City, China. Tel: \\ 187-8018-1027. E-mail: 18780181027@163.com
}

Received: August 27, 2015

Accepted: September 25, $2015 \quad$ Online Published: November 25, 2015

doi:10.5539/ijef.v7n12p253

URL: http://dx.doi.org/10.5539/ijef.v7n12p253

\begin{abstract}
Industrial Structure Change is not only an important source of economic growth; it's also an important driving force for economic fluctuations. In this paper, on the basis of combing the literature, and the relevant data in 1978-2013 of Sichuan Province in China, and the use of empirical VAR model to analyzes the mutual dynamic influence of Sichuan Industrial Structure Change and economic fluctuations. The study found that the rationalization and optimization of the industrial structure both impact on economic fluctuations, but the impact are on the opposite direction. In the short term, the industrial structure rationalization and industrial structure optimization respectively has positive and negative effects on economic fluctuations; in the long term the opposite. Industry Structure optimization fluctuations shows a great influence on economic fluctuations, while the rationalization of the industrial structure shows a relatively small negative effect. The impact of economic fluctuations on the rationalization of the industrial structure fluctuation and optimization performance for the negative and positive relationships.
\end{abstract}

Keywords: industrial structure, economic fluctuations, VAR model

\section{Introduction}

Industrial Structure Change is an important source of economic growth, is a process of economic growth of technological innovation absorbing and leading industry sectors' sequential replacement. Thus brought the flowing of elements in different industrial productivity levels, and promote the increase of the whole society productivity levels. At the same time, the Industrial Structure Changes will also bring some problems in promoting industrial development, and sustaining of economic growth. On the one hand, technological progress and technological alternative impact would break the original economic balance, so that changes in the supply of certain factors of production sectors, thus causing economic fluctuations. On the other hand, the leading industrial policy will cause changes in the structure of social investment and consumption structure, and thus affect the stability of the economy (Chunhui Gan et al., 2011). Therefore, change in the industrial structure is one of the main driving forces of the economic cycle (Kuznets, 1971).

Yanbin Chen (2005) pointed out that the contribution of the welfare costs of economic fluctuations and economic growth brought to China are roughly equivalent, the national economy has paid a great price, therefore, economic development requires sustained and healthy development. "The Twelfth Five-Year Plan" pointed out that to correctly handle the relationship of steady economic development, economic restructuring and management of inflation expectations, guard against all kinds of potential risks, and to avoid ups and downs of the economy. "The Eighteenth General Assembly People's Congress" stressed the need to focus on optimizing the industrial structure to accelerate the strategic adjustment of economic structure. At the same time, Sichuan "Twelve Five Year Plan" showed the necessary of vigorously readjust the industrial structure optimization and upgrading, three industrial structure adjustment of 10.2: 50.8: 39, the urbanization rate reached about $48 \%$ by 2015. So, exploring the relationship between industrial structure change and economic fluctuation has great significance of the adjustment of industrial structure to promote economic growth while maintaining steady economic development for Sichuan Province. In the study of industrial structure change and economic fluctuation, Eggers and Ioannidis (2006) through the study in 1950 to the United States found that the contribution ratio of the Industrial Structure Change on economic fluctuations stabilizing trend is up to 50\%, noting that the existing literature underestimates of effect on the industrial structure transition. Accordingly, 
Meng Li et al. (2010) analyzed the impact of Chinese economic fluctuation source and impact of industrial structure, and found the impact of the industrial structure contributes about $15 \%$ to 20\%. Xiahui Liu (2004) concluded that, economic fluctuation with the economic growth is a significant feature of China's economic development. Jiang Yuan, Chengsi Zhang (2009) further explained that in each economic cycle, the total economy and the economic expansion will be accompanied by structural differentiation, conversely, when the economic downturn, the unbalanced of economic structure shrinking. From each industry's cycle-fluctuations and the impact of economic fluctuations, Guangsheng Sun (2006) considered th secondary industry and macroeconomic volatility has strongest correlation, followed by the tertiary industry, and the first industry is not relevant. Yune $\mathrm{Li}$ (2008) found that the second industry and the first industry have greatest contribution to macroeconomic fluctuations, there is no causality between the tertiary industry and macroeconomic fluctuations, macroeconomic volatility is not the root cause of industrial restructuring. Yu Wang et al. (2011) based on structures catastrophe model of Bayesian method showed that second industry plays a decisive impact on the cyclical fluctuations of the conclusion of China's economic growth. Fuqian Fang, Xinyu Zhan (2011) used TGARCH model to found that three major industries have asymmetric effects on the economy fluctuations in China.

Otherwise,many scholars measure the industrial structure changes to study its' effect and contribution to economic fluctuations. Peng Chong et al. (2013) pointed out that rationalization of industrial structure exhibits weak counter-cyclical impact of economic fluctuations, it is a "smoothing effect", and upgrading fluctuations impact is strongly pro-cyclical. Li Qiang (2012) found that the industrial structure rationalization and advancement will help alleviate the economic fluctuations, but the impact of upgrading effect is more significant. Similarly, Chunhui Gan (2011) also believes that upgrading of the industrial structure is an important source of economic fluctuations, the industrial structure rationalization can help alleviate the economic fluctuations. Yanjun Wang (2011) adopted structural change value K and Moore index as measure of three industrial structure changes, the analysis found that economic fluctuations is the Granger cause changes in the industrial structure, otherwise not.

In summary, the study of industrial structure change and economic fluctuations mainly focus on the national level. But in China, there are differences in natural, historical, cultural and other presence in different province. So, the relationship between industrial structure change and economic fluctuations is bound with geographical features, and due to the selection of indicators, each conclusion may be a little different. Also, there are fewer study of two-way dynamic affect between industrial structure changes and economic fluctuations within the study area. Therefore, this paper chooses Sichuan Province as the research object, and the selection of the indicators referencing many scholars in points and views, from two angles of the rationalization and advancement of the industrial structure, to analysis the two-way dynamic effects between industrial structure change and economic fluctuations.

\section{Research Methods, Variable Selection and Data Description}

\subsection{Research Methods}

In this paper, the vector auto regression model (VAR models) is used to analyze the relationship between industrial structure and economic growth. VAR model proposed by the Sims in 1980, it sets each endogenous variable in the system as a function of the system lagged values of all the endogenous variables to construct the model, extending the univariate autoregression model to the multivariate time series variable, composition of the "vector" Since the regression model. Lag of P's mathematical expression for the VAR(p) model are:

$$
Y_{t}=c+A_{1} Y_{t-1}+A_{2} Y_{t-2}+\cdots+A_{p} Y_{t-p}+\mathrm{h} X_{t}+\varepsilon_{t}, \mathrm{t}=1,2, \cdots, \mathrm{T}
$$

$\mathrm{K}$ represents an endogenous variable dimension column vector, $\mathrm{X}_{\mathrm{t}}$ is the D-dimensional column vector of exogenous variables, $P$ is the lag order, $T$ is the number of samples. $k \times k$ Dimensional matrix $A_{1}, A_{2} \ldots A_{P}$ and $\mathrm{H}$-dimensional matrix $\mathrm{k} \times \mathrm{d}$ is to be estimated coefficient matrix. $\varepsilon_{\mathrm{t}}$ is a k-dimensional perturbation vector.

\subsection{Data Sources and Variables Selection}

\subsubsection{Data Sources}

This paper considering the data availability and effectiveness, and selecting data from 1978-2013 of Sichuan Province, and the sample data mainly obtained directly or calculated from the "Sichuan Statistical Yearbook 2014" and "six years statistics compilation new China of new China". 


\subsubsection{Variables Selection}

For the method of measuring fluctuations, the more commonly used methods include time trend method, first-order differential method, HP filter method and BP filter. HP filter has its excellent properties, and has been very good application, this paper intends to use HP filter methods to measur fluctuations of economic variables, and selecting the following variables as explanatory variables and explanatory variables. Setting economic fluctuations as the dependent variable (YO), firstly, setting 1978 as the base period, to carry out each year's GDP deflator, and then get data through the HP filter. Independent variable include the industrial structure rationalization gap (TLO) and the Upgrading of the Industrial Structure notch (TSO), obtained from the same HP filtering of rationalization index and high-class index. Due to the use of annual data, in accordance with the recommendations of Ravn \& Uhlig and other scholars, the smoothing parameter in this paper is set to 100 .

\subsection{Industrial Structure Change Index Measure}

Many scholars believe that the industrial structure optimization and upgrading is actually the rationalization and advancement of industry structure. Therefore, this paper intends to measure the dynamic processes of industrial structure changes through the two dimensions.

\subsubsection{Industry Structure Rationalization Index}

Currently, scholars have not yet drawn up a unified standard on the selection of indicators industrial structure rationalization measure. They build measure index by relevant theory according to their actual needs. This paper agree with Chunhui Gan's (2011) view: on the one hand, industry structure rationalization is a reflection of the degree of coordination between industry; on the other hand, it is a measure of the degree of coupling about elements and resource allocation efficiency and the output structure. So, this paper use metrics of rationalization industrial structure:

$$
T L=\sum_{i=1}^{n}\left(\frac{Y_{i}}{Y}\right) \ln \left(\frac{Y_{i}}{L_{i}} / \frac{Y}{L}\right)
$$

$\mathrm{Y}, \mathrm{Y}_{\mathrm{i}}, \mathrm{L}, \mathrm{L}_{\mathrm{i}}$ represents regional production, the three industrial output value, total employment, and employment in the three industries. According to the definition, the lower the industrial structure rationalization index is, the higher degree of its rationalization, and vice versa.

\subsubsection{Industrial Structure Optimization Index}

As the measure for optimization of Industrial Structure, different scholars have their own view. Among the many studies, more accepted view is that optimization of industrial structure is mainly the product mix goes to higher value-added industrial evolution, departmental structure goes to technology-intensive and knowledge-intensive industrial research, especially the development of information technology, and the industrial structure trend to a "service economy". Therefore, this paper think that the economic services is trend of the modern high-class economic structure. Referencing Chunhui Gan's practices, this paper use the ratio of tertiary industry output value and the second industry output (TS) to measure the optimization of industry. The higher index means the higher degree of industrial structure optimization.

\section{Empirical Analysis}

\subsection{Descriptive Statistics}

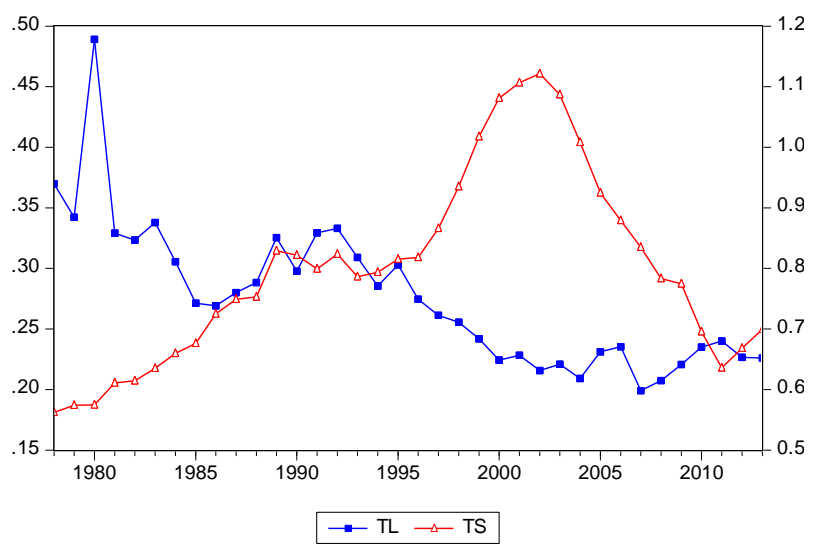

Figure 1. Rationalization and optimization index of sichuan province in 1978-2013 
As it can be seen from Figure 1, since the reform and opening up the rationalization index of industrial structure in Sichuan tends to decrease significantly, indicating the industrial structure of Sichuan is in the process of constantly adjust, the ratio and the mass coupling among the three major industries have been continuously optimized. But in the adjustment process, showing a different rate fluctuation, the largest volatility is in 1980, it is related to China's rural household responsibility system, which is a pioneer in Sichuan rural reform, and has greatly promoted the rural economy development. Optimization index of Sichuan has been on the rise until 2002, and it rose significantly faster in 1997 . This is because this paper use the ratio of the tertiary industry output value and the second industry output value, and Chongqing city became a municipality, leading the second industry in Sichuan declined to some extent, so high-class industrial index showed a clear upward trend. Coupled with the impact of the Asian financial crisis in 1997 also have a greater impact on the province's industrial entities economic. In 2002, the industrial optimization index decreased rapidly, indicating that Sichuan Province was developing industry. Especially the implementation of the western development promoted the rapid development of Sichuan industrial base and the second industry, as the economic center in western China, economic strategy of Sichuan Province has been to take a new road to industrialization in recent years, and attracting foreign investment, the second industry shows rapid growth. And the national comparison shows in 2010 the degree of rationalization of the industrial structure in Sichuan was at the middle level in the country, however, the degree of industry structure optimization lag behind in the country, still in the acceleration phase of industrialization.

Further examining the relationship between industrial rationalization and advancement and economic volatility, we put 2000 western development as a demarcation point to divided 1978-2013 into two stages to describe the economic variables.

Table 1. Periodic fluctuations feature of major economic variables

\begin{tabular}{lrrrrrr}
\hline Economic & \multicolumn{3}{c}{ Standard Deviation } & \multicolumn{3}{c}{ Correlation Coefficient Economic Fluctuations over the same period } \\
\cline { 2 - 7 } variables & $1978-1997$ & $1998-2013$ & $1978-2013$ & $1978-1997$ & $1998-2013$ & $1978-2013$ \\
\hline TLO & 0.04 & 0.01 & 0.03 & 0.06 & 0.16 & 0.08 \\
TSO & 0.04 & 0.06 & 0.05 & -0.69 & -0.70 & -0.65 \\
Y0 & 28.79 & 21.11 & 25.69 & 1.00 & 1.00 & 1.00 \\
\hline
\end{tabular}

As we can see from the volatility, the economic fluctuation was higher than rationalization and optimization of industry structure. Specifically, economic fluctuations after 2000 was significantly lower than the level before 2000.It shows that Sichuan seized the opportunity to develop industry and the anti-volatility of economy significantly enhanced. The volatility of rationalization of industry structure in 2000 was lower than before, due to the vigorously readjust of the industrial structure in Sichuan. However, The volatility of optimization of industry structure has a slight rise after 2000. From the correlation coefficient of output volatility, the correlation coefficient of rationalization industry structure and economic fluctuation was weak positive in 1978-2013, it showed weak cyclical before 2000 and a strong pro-cyclical after 2000. In the same time, the correlation coefficient of optimization industry structure and economic fluctuation was relatively large, and showed counter-cyclical both before and after 2000.

\subsection{Stationary Test of Variables}

To avoid the "spurious regression" phenomenon caused by unstable data, we use ADF test method to test for the stability of the variables, the results shown in Table 2. The is that economic fluctuations index, rationalization index of industry structure and optimization fluctuations index of industry structure rejected the unit root null hypothesis at the 5\% significance level, so the variables are stationary.

Table 2. variable unit root test results

\begin{tabular}{crccc}
\hline variables & Test forms $(\mathrm{C}, \mathrm{T}, \mathrm{K})$ & ADF statistics & $5 \%$ critical value & Result \\
TLO & $(\mathrm{C}, 0,0)$ & -5.969449 & -2.948404 & stationary \\
TSO & $(\mathrm{C}, 0,3)$ & -4.618265 & -2.957110 & stationary \\
YO & $(\mathrm{C}, 0,1)$ & -3.161995 & -2.951125 & stationary \\
\hline
\end{tabular}

\subsection{VAR Model Estimates}

Determine the maximum lag $\mathrm{K}$ is very important before the establishment of VAR model. The criterion of the lag 
order is variety, including LR statistics, Akashi information criterion (AIC) and Schwarz criterion (SC). As Table 3 shows the lag period of 2 has four remarkable statistic, and the rest have no significant lag, so we can determine the lag period is 2 , and establish VAR model.

Table 3. Model selection at K lag

\begin{tabular}{cccccc}
\hline Lag & Log & LR & FPE & AIC & SC \\
\hline 0 & -7.9285 & NA & 0.000397 & 0.683031 & 0.820444 \\
1 & 29.01039 & $64.64306^{*}$ & $6.96 \mathrm{e}-05^{*}$ & $-1.063150^{*}$ & $-0.513499^{*}$ \\
2 & 37.56739 & 13.3703 & $7.28 \mathrm{E}-05$ & -1.03546 & -0.07357 \\
3 & 43.26045 & 7.827963 & $9.35 \mathrm{E}-05$ & -0.82878 & 0.545349 \\
4 & 53.36329 & 11.99712 & $9.49 \mathrm{E}-05$ & -0.89771 & 0.88866 \\
\hline
\end{tabular}

The VAR model is estimated as follows:

$$
\begin{aligned}
\left(\begin{array}{c}
Y O \\
T S O \\
T L O
\end{array}\right)=A *\left(\begin{array}{c}
Y O \\
T S O \\
T L O
\end{array}\right)_{t-1}+B *\left(\begin{array}{c}
Y O \\
T S O \\
T L O
\end{array}\right)_{t-2}+C \\
A=\left(\begin{array}{rrr}
0.796562 & -116.8393 & 65.96886 \\
0.000342 & 1.197862 & -0.051463 \\
0.000179 & -0.277966 & -0.125410
\end{array}\right) \\
B=\left(\begin{array}{rrr}
-0.386625 & 6.57 \mathrm{E}-05 & 0.000252 \\
-52.29101 & -0.383456 & 0.416448 \\
94.34686 & -0.248468 & -0.041493
\end{array}\right) \\
C=\left(\begin{array}{r}
-1.144554 \\
0.001571 \\
0.001514
\end{array}\right)
\end{aligned}
$$

As it can be seen from Figure 2, VAR model has good stability. Based on this, it is credible to analyze the dynamic effects of rationalization and optimization of industry structure with economic fluctuations.

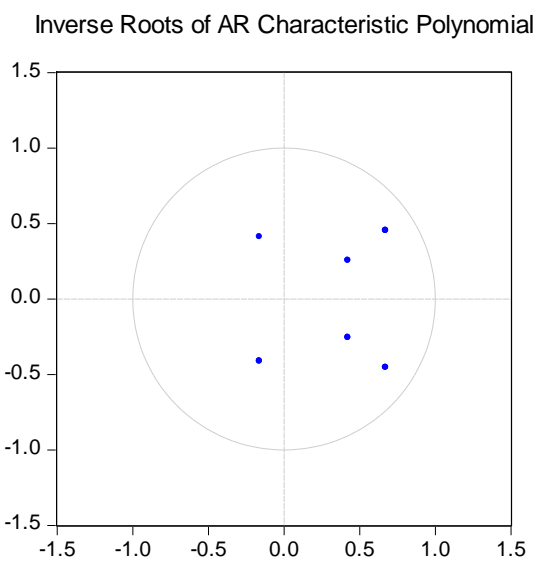

Figure 2. Model stability test

\subsection{Impulse Response Function}

Impulse response function shows the impact on current value and future value arising endogenous variables cause $\mathrm{d}$ by adding one standard deviation shock onto the disturbance term. In Figure 3, giving one standard deviation shock to TLO, immediately YO will generate a weak positive impact and it gets maximum in the third period, and then followed by a positive effect gradually weakened, and switch slight into negative effect until 
after 8 periods, it shows that volatility of industry structure rationalization and economic fluctuations positive relationship in Sichuan Province. When giving a standard deviation shock to TSO, YO then generates a gradually increasing negative effect, and reached the maximum in the first four period, and then decreases until after the seven, turning positive impact. Therefore, rationalization volatility of industrial structure in Sichuan has a positive impact on the early economic fluctuations, while optimization volatility of industrial structure have a greater negative impact on economic fluctuations, this conclusion is in contrast with Chong Peng's research in the entire country.

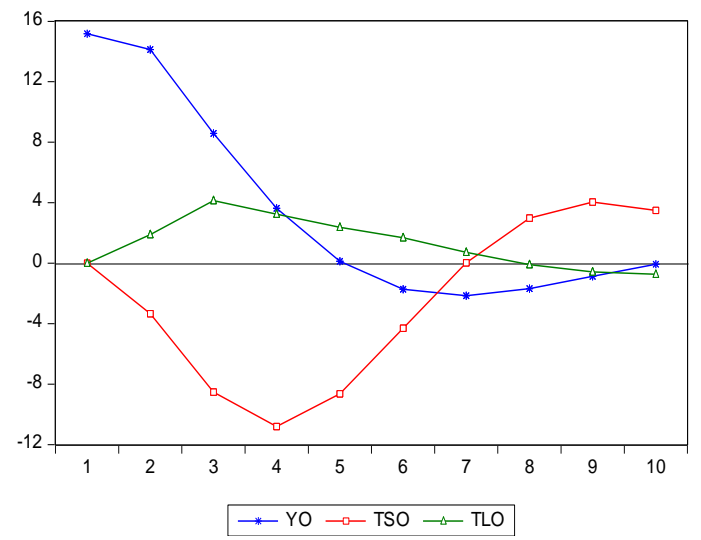

Figure 3. YO's response to shocks

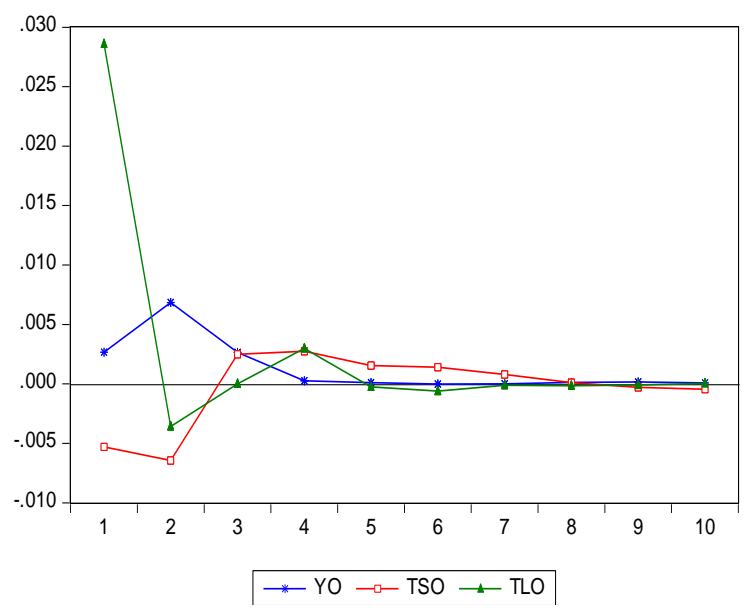

Figure 4. TLO's response to shocks

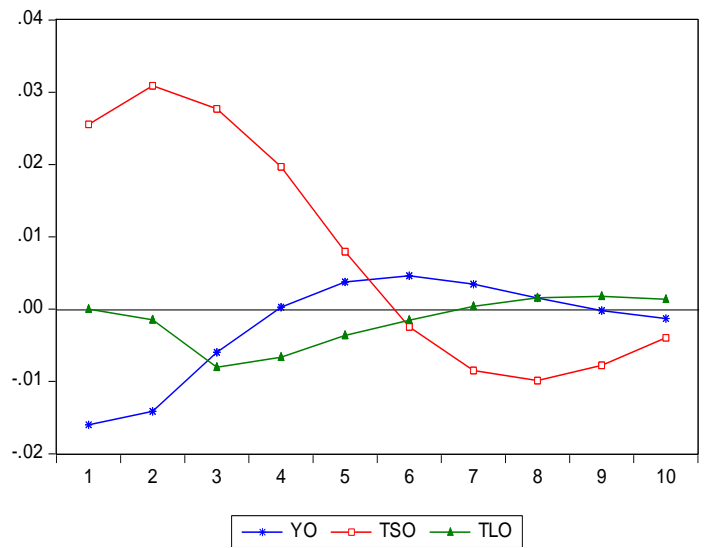

Figure 5. TSO's response to shocks 
From Figure 4, it can be found that he impact of optimization of industrial structure on economic fluctuations will turn positive influence, which is consistent with the conclusions of Peng Chong (2011). One of the reasons may be the vary of the selected measure indicators, but on the other hand, it also shows that Sichuan Province has its own characteristics compared with the national, and this is precisely the key to this paper. Overall, the impact of the rationalization of the industrial structure has a greater positive effect on economic fluctuations, weakening in the long run and turning into a negative impact in the end; while the impact of the optimization of the industrial structure has a negative impact on economic fluctuations in the short term, and shows a positive impact in long term, this might be Sichuan is vigorously restructuring, and industries to relocate from eastern area. Due to industrial development, Sichuan's economic shows rapid growth, but it is subject to international and domestic situation, showing volatility at the same time. Sichuan is developing the information technology industry and the feature of consumer entertainment itself has contributed to the rapid development of the tertiary industry, therefore, exhibiting different characteristics in the short term, but it will return to the normal track in the long run.

As we can see from Figure 4, when giving one standard deviation shock to YO, TLO first exhibited positive impact, then tends to zero, resulting in a weak alternating positive and negative impact after four period. Therefore, the rationalization of industrial structure has a weak positive effect on economic fluctuations. The TSO shows negative effects, the initial value is large negative value, weakened rapidly in the short term, turning positive impact after four periods, but the positive impact is relatively small, and gradually weakened, and switching negative impact direction in the first 9 period. Overall, the economic fluctuations has a slight weakening positive influence of the industrial structure rationalization fluctuations in advance, and there is a weak alternating positive and negative impact in the long term, while the Industrial Structure fluctuations from negative to a positive and then negative impact, while has an impact on optimization of industrial structure from negative to positive and then a negative. To the shocks from TLO, the TSO first shows a negative effect, random into small positive effect, but turns into a weak negative effect after the first eight period, but the shock from TSO to TLO has shown a negative reaction at 1-7 period, then converts to a positive response, tends to zero. Overall, the optimization of industrial structure has a negative impact on the industrial structure rationalization process in the short term, and has a positive impact at medium term, and the long-term performance is a weak negative effect. While the industrial structure rationalization has a large impact on optimization of industrial structure, it is negative in short term, and positive in long term.

In summary, the shocks from rationalization and optimization of industrial structure both have an impact on economic fluctuations, but the direction is opposite. Rationalization of the industrial structure has a positive impact on the economic fluctuations in the short term, have a negative effect in long term, while optimization of industrial structure has a large negative effect on the economic fluctuations in the short term, a positive impact in long term. In turn, the economic fluctuations has a positive impact on the industrial structure rationalization volatility in the short term, and a weak alternating positive and negative impact in the medium and long term; while has a weakened negative impact on optimization volatility of industrial structure in short term, a positive influence in the medium-term, and turns into negative in the long-term impact.

\subsection{Variance Decomposition}

In order to describe and examine the degree of influence between industry structure change and economic volatility more accurately, we use variance decomposition method to obtain the variance contribution degree of economic volatility from the impact of different VAR equation, variance decomposition results are shown in Table 4.

Rationalization fluctuation of industry structure variance has its own contribution degree at 84.89 percent, contribution from optimization of industrial structure and economic fluctuations are $6.17 \%$ and $8.95 \%$. So the fluctuations are mainly from the impact itself, the system tend to be stable in the 10th period. Optimization fluctuations of industrial structure in addition to get shocks from its own, but also affected by economy fluctuations as the variance Contribution degree is $14.46 \%$, while the contribution of the industrial structure rationalization fluctuation is $3.57 \%$. 
Table 4. Variance decomposition

\begin{tabular}{|c|c|c|c|c|c|c|c|c|c|}
\hline \multirow{2}{*}{$\begin{array}{c}\text { Forecast } \\
\text { Period }\end{array}$} & \multicolumn{3}{|c|}{ TLO variance decomposition $(\%)$} & \multicolumn{3}{|c|}{ TSO variance decomposition $(\%)$} & \multicolumn{3}{|c|}{ YO variance decomposition $(\%)$} \\
\hline & TLO & $\mathrm{TSO}$ & YO & TLO & TSO & YO & TLO & TSO & YO \\
\hline 1 & 95.88 & 3.29 & 0.83 & 0.00 & 71.84 & 28.16 & 0.00 & 0.00 & 100.00 \\
\hline 2 & 87.07 & 7.29 & 5.64 & 0.10 & 77.83 & 22.06 & 0.80 & 2.50 & 96.70 \\
\hline 3 & 85.88 & 7.83 & 6.30 & 2.28 & 80.97 & 16.75 & 3.40 & 13.79 & 82.82 \\
\hline 4 & 85.34 & 8.46 & 6.20 & 3.29 & 82.10 & 14.60 & 4.17 & 26.79 & 69.05 \\
\hline 5 & 85.14 & 8.68 & 6.18 & 3.59 & 81.78 & 14.63 & 4.44 & 33.20 & 62.36 \\
\hline 6 & 84.97 & 8.86 & 6.17 & 3.63 & 81.26 & 15.11 & 4.65 & 34.42 & 60.93 \\
\hline 7 & 84.91 & 8.92 & 6.16 & 3.55 & 81.36 & 15.10 & 4.68 & 34.21 & 61.10 \\
\hline 8 & 84.91 & 8.92 & 6.17 & 3.51 & 81.75 & 14.74 & 4.62 & 34.77 & 60.61 \\
\hline 9 & 84.90 & 8.93 & 6.17 & 3.54 & 81.97 & 14.49 & 4.57 & 35.94 & 59.50 \\
\hline 10 & 84.89 & 8.95 & 6.17 & 3.57 & 81.97 & 14.46 & 4.56 & 36.78 & 58.66 \\
\hline
\end{tabular}

Compared with the optimization of industrial structure, the rationalization industrial structure has a smaller impact on economic volatility, only $4.56 \%$. From the beginning, the rationalization and optimization of the industrial structure impact on economic fluctuations both at the contribution degree of zero, but the growth rate of optimization is faster than the industrial structure rationalization, eventually reaching $36.78 \%$. This is mainly because of the major adjustment of the industrial structure caused by implementation of the western development strategy and deepen openness. Chengdu city is the growth pole of Sichuan Province, largely polarized resources of other cities and prefectures in Sichuan. Due to the rapid development of the secondary industry, and the tertiary industry has also entered the fast lane, especially the creation of western logistics center, the construction and adjustment of information industry park and high processing technology, making the industrial structure with a relatively large degree of high-class upgrade. However, the transportation, raw materials and other basic industries can not meet the needs of other industries, on the one hand, it causes to rationalization fluctuations of industrial structure, on the other in hand, it accelerates the optimization volatility of industrial structure.

\section{Conclusions and Recommendations}

\subsection{Conclusions}

It has been confirmed by many scholars that structural changes is an important source of economic fluctuations are, but relatively speaking ,there are still less support experiences, most studies are on the national macro-level, but ignoring that each province have its own characteristics because of differences in natural, historical and cultural conditions, and also in some respects must. Therefore, this paper learns from relevant indicators of other scholars, and use rationalization gap of industrial structure and optimization gap to measure the fluctuation, and the VAR model is sued to analyze the dynamic effects of economic fluctuations in Sichuan Province.

Industry structure rationalization and optimization both have impact on economic volatility, but the impact direction is opposite. In the short term, the industrial structure rationalization and industrial structure optimization respectively has positive and negative effects on economic volatility; and the opposite in the long term. However, Economic volatility has a positive impact on rationalization volatility of industrial structure in Sichuan in the short term, with a weak alternating positive and negative impact in the long term; while economic volatility has a decrease negative effect on optimization volatility of industrial structure in a short term, a positive influence in the medium term, and turns into negative in the long term.

As the results from variance decomposition, the rationalization volatility of the industrial structure in Sichuan shocks mainly by itself; optimization volatility of industrial structure in addition to shocks by itself, economic fluctuations is also an important factor, but impact from the industrial structure rationalization fluctuations is small.

\subsection{Recommendations}

Firstly, promoting the optimization and upgrading of infrastructure and basic industries. Road and transport infrastructure construction and upgrading are bottleneck factors which could constrain optimization of industrial structure of and cause volatility. The optimization of industrial structure is an important source of economic fluctuations, in the short term has a so-called screed effect, but in the long run shows a positive impact on the economy, therefore the fluctuations has an incubation period, so, the province should adjustment policies based on in the development of industry in a long-term perspective, and take into account fluctuations of industrial Structure optimization. 
Secondly, optimizing the industry structure, vigorously develop the strategic emerging industries and producer services. Although the rationalization of the industrial structure has a positive impact on Economic volatility in short term, but its impact is relatively small, and the impact turns to a negative direction in the long run. So, do not be afraid to economic volatility brought by adjusting the industrial structure at this stage, optimizing the share proportion among the three major industries continue to consolidate the basic position of the first industries, taking a new road to industrialization, vigorously developing strategic emerging industries and productive services, combining the reverse affect from the industrial structure rationalization and optimization, and jointly promoting the stable and healthy economic sustainable development in Sichuan Province.

Thirdly, introducing foreign investment, and taking a new road to industrialization. Currently, the Sichuan province is in the acceleration phase of industrialization, it is necessary to develop strategic emerging industries, but should not excessive pursuit of high-class industrial structure, because of the rationalization of the industrial structure industrial structure is the foundation of the optimization of industrial structure. Therefore, promoting high-class should base on the rationalization of the industrial structure, formulating relevant industrial policies to create a fair market environment. Vigorously to attract foreign investment and ready to undertake the industry transfer of the eastern area.

Finally, thanks for my mentor's advising, and my classmate who help me use the VAR model smoothly. In the next step, I will conduct more intensive research.

\section{References}

Fuqian, F., Xinyu, Z., \& Smoothing. (2011). Effect analysis of Chinese industrial structure upgrading to economic fluctuations. Economic Theory and Business Management. http://dx.doi.org/10.3969/j.issn.1000-596X.2011.09.001

Linghui, F. (2010). Empirical Study on Industrial Structure Upgrade and Economy Growth. Statistical Research. http://dx.doi.org/10.3969/j.issn.1002-4565.2010.08.011

Simon, K. (1971). Economic Development, the Family and Income Distribution: Selected Essays. Cambridge University Press.

Yanbin, C. (2007). The Welfare Analysis of the Predictability of Business Cycles in China. Economic Theory and Business Management. http://dx.doi.org/10.3969/j.issn.1000-596X.2007.02.002

Yanbin, C., \& Yimin, Y. (2010). Sources of China's Economic Growth: 1978-2007. Economic Theory and Business Management.

\section{Copyrights}

Copyright for this article is retained by the author(s), with first publication rights granted to the journal.

This is an open-access article distributed under the terms and conditions of the Creative Commons Attribution license (http://creativecommons.org/licenses/by/3.0/). 